\title{
FREQUENCY AND ANTIMICROBIAL SUSCEPTIBILITY PATTERN OF PSEUDOMONAS AERUGONISA CLINICAL ISOLATES AT A LARGE TEACHING HOSPITAL IN LAHORE, PAKISTAN
}

\author{
SARMAD ZAHOOR, HAFIZ MUDABBAR MAHBOOB, HINA BUKHARI, ASAD ALI, \\ HAFIZ MUHAMMAD SAJID JEHANGIR, ARSHAD SIDDIQUI \\ King Edward Medical University, Mayo hospital, Lahore
}

\begin{abstract}
Background: Psudomonas is a notorious nosocomial aerobic bacillus mostly involved in causing pneumonia, surgical site infection and urinary tract infection. Resistance of pseudomonas has increased in recent past and has posed a serious health challenge to public health.

Objective: To determine resistance pattern of Pseudomonas aeuroginosa from clicical isolates at Mayo Hospital, Lahore during 2019.

Methods: This was a Single Centre Observational Cross-Sectional Retrospective Study conducted at Mayo hospital Lahore which included clinical isolates of pseudomonas isolated during 2019 obeying inclusion and exclusion criteria. Organism was identified with standard protocols and was tested for susceptibility to various antibiotics as per CSLI guidelines. Data was analyzed with SPSS 23.

Results: Of 1506 isolates, the most sensitive drug was collistin (100\%). Relatively higher sensitivity was reported for imipenum (94.48\%) followed by amikacin $(91.96 \%)$. Least sensitive of all the tested drugs was ceftriaxone $(12.48 \%)$ followed by tazobactum/pipperacillin (33.3\%). Ceftazidime (74\%), gentamycin (70.1\%) and ciprofloxacin $(65.6 \%)$ showed intermediate level of sensitivity in our study.

Conclusion: Collistin, Imipenum and amikacin are the effective drugs against pseudomonas. However, the resistance of the bug is increasing which warns for judicious use of antibiotics.
\end{abstract}

Key Words: Pseudomonas, Antibiotics, Sensitivity, Resistance

How to cite this article: Zahoor S, Mahboob HM, Bukhari H, Ali A, Jehangir HMS, Siddiqui. Frequency and antimicrobial susceptibility pattern of pseudomonas aerugonisa clinical isolates at a large teaching hospital in Lahore, Pakistan. Pak Postgrad Med J 2020;31(4): 168-171

This is an Open Access article distributed under the terms of the Creative Commons Attribution License (http://creativecommons.org/licenses/by/3.0), which permits unrestricted use, distribution, and reproduction in any medium, provided the original work is properly cited.

DOI: http://doi.org/10.51642/ppmj.v31i04.366

Correspondence to: Sarmad Zahoor,

Department of Pathology, King Edward Medical

University, Mayo hospital, Lahore, Pakistan.

Email: drsarmadzahoor@gmail.com

\section{INTRODUCTION}

Anti-Microbial Drugs are the mainstay of treatment against bacterial Infections. Advancement in the field of antibiotics has resulted in saving millions of lives. However, Emergence of antibiotic Resistance is a global threat and a cause of concern for mankind ${ }^{1-2}$. There are several factors responsible for this much increase in antibiotic resistance. These includes misuse of antibiotics, lack of awareness both by physicians and public, and self medication ${ }^{3-4}$.

Pseudomonas Aerugonisa (a member of ESKAPE group that includes Enterococcus faecium, Staphy-lococcus aureus, Klebsiella pneumoniae, Acinetobacter baumannii, Pseudomonas aeruginosa and Enterobacter), a gram-negative aerobic bacillus, is among the one of the emerging pathogen of group and is involved in diverse variety of nosocomial infections especially pneumonia, urinary tract infection, surgical site infections and bacteremia $^{5-6}$. Pseudomonas has contributed greatly in the mortality of hospitalized patients ${ }^{7}$. This is surely due to its ability to easily develop resistance against antibiotics through several mechanisms. These includes 
production of lactamases, carbapenamases, over expression of efflux pump and Amp C-Lactamases, change in target site and a type of unstable resistance which is acquired by the exposure of broad-spectrum antibiotics i.e due to antibiotic pressure ${ }^{8-10}$.

Several antipseudomonal drugs have conventionally been used against pseudomonas which are fluroquinolones (ciprofloxacin), cephalosporin (ceftazidime, cefepime), antipseudomonal pencillins (Tazobactum,Pipperacillin), Aminoglycosides (amikacin \& gentamycin) and carbepenam (imepenam and meropenam) ${ }^{11}$. Over the last decades carbepenams has been considered as drug of choice. Since last few years, resisitance to carbepenam has been repoterd ${ }^{12}$. To cope with the problem, colistin is used in multi-drug resistant (MDR) pseudomonas as a last resort ${ }^{13}$. However, Colistin Resistance has also been documented in the recent past ${ }^{14-15}$. Resistance pattern of an organism is very variable. It changes with time and place ${ }^{16}$.

So, the need of the hour is such a study describing susceptibility pattern of the bug. In our search of the literature, no study is available showing road map of resistance for pseudomonas in Pakistan for 2019.We have conducted such study elaborating susceptibility of recent isolates of pseudomonas at a large teaching hospital of Lahore.

\section{METHODS}

It was an observational cross-sectional retrospective study conducted at Microbiology section, Pathology department of King Edward, Medical University, Mayo Hospital, Lahore after permission of ethical review board of the institution. All the isolates of pseudomonas during year 2019 i.e 1506, which showed positive growth and were tested for susceptibility of antibiotics were included in the study. Whereas all those samples Which were either negative for pseudomonas or were not tested for antibiotics were excluded from the study.

All the samples obtained either from ward or OPD were cultured according to standard guidelines. Further confirmation was done using special biochemical tests and ApI (Biomerieux). After the separation, antibiotics susceptibility analysis was made following CSLI (Clinical and Laboratory Standards Institute) guidelines ${ }^{17}$.

Data was analyzed using SPSS version 23.0. Qualitative statistics will be determined as frequency and percentages.

\section{RESULTS}

A total of 1506 (24\%) stains of pseudomonas were isolated from all the culture growths (6280) during 2019. Among these 883(58.63\%) were females and 623 $(41.37 \%)$ were male. Most of the samples were obtained from wound swab (524) and pus (439) followed by tacheobronchial secretions (268), sputum (97), blood culture (90), catheter tip (78) and chest tube aspirate (10). Relative Distribution of sources with their percentages is shown below in the table:

Table 1: Distribution of the Source of Specimen

\begin{tabular}{lc}
\hline Source & Pseudomonas frequency \\
\hline Wound Swab & $524(34.79 \%)$ \\
Pus & $439(29.15 \%)$ \\
Trachebronchial Secretions & $268(17.8 \%)$ \\
Sputum & $97(6.44 \%)$ \\
Catheter tip (CVP and ETT) & $78(5.18 \%)$ \\
Blood Culture & $90(5.98)$ \\
Chest Tube aspirate & $10(0.66 \%)$ \\
\hline
\end{tabular}

Pseudomonas was seen to be resistant to almost all the anti-pseudomonal antibiotics. Colistin was the only drug which was $100 \%$ sensitive and no resistance was seen. Highest resistance was observed with ceftriaxone $(87.52 \%)$ The sensitivity of Imipenem, ceftazidime, Amikacin, ciprofloxacin, gentamycin and tazobactumpipperacillin was $94.48 \%, 74.1 \%, 91.96 \%, 65.6 \%$, $70.18 \%$ and $33.33 \%$ respectively. The Distribution of resistance and sensitivity pattern of all the isolates of Pseudomonas with their relative percentages is given below:

Table:2 Susceptibility pattern of Pseudomonas Aeruginosa

\begin{tabular}{lcc}
\hline Antibiotics & Sensitive & Resistance \\
\hline Ceftazidime & $1116(74.1 \%)$ & $390(25.9 \%)$ \\
Tazobactum- & $502(33.33 \%)$ & $1004(66.67 \%)$ \\
Pipperacillin & & \\
Imipenam & $1423(94.48 \%)$ & $83(5.52 \%)$ \\
Ceftriaxone & $188(12.48 \%)$ & $1318(87.52 \%)$ \\
Ciprofloxacin & $988(65.6 \%)$ & $518(34.4 \%)$ \\
Amikacin & $1385(91.96 \%)$ & $121(8.04 \%)$ \\
Gentamycin & $1057(70.18 \%)$ & $449(29.82 \%)$ \\
Colistin & $1506(100 \%)$ & $0(0 \%)$ \\
\hline
\end{tabular}

\section{DISCUSSION}

Pseudomonas has been implicated in causing many hospitals acquired infections especially in immunecompromised individuals. To treat it, is a big challenge and a major concern for health care providers since its resistance has increased in recent past. This needs to be addressed vigilantly to have a better outcome ${ }^{18}$.

The most frequent source of sample was wound and pus with other minor sources being blood, catheter tip, tarachebronchial secreations and chest tube aspirate which is consistent with other studies ${ }^{19}$. Another source 
was sputum which was $6.4 \%$ of all positive cultures which is in contrast to other studies ${ }^{20-21}$.

In the context of resistance and sensitivity pattern, highest resisitant drug was ceftriaxone $(87.5 \%)$ and tazobactum /pipperacillin (66.67\%). This high resistance for tazobactum/pipperacillin is highly alarming and is likely contributed due to its frequent use.This is in accordance with other local studies and was higher in percentage to an international study reported by Micek et al. ${ }^{22-25}$. Ciprofloxacin showed better results and was resistant in $34.4 \%$ cases only. Ceftazidime was sensitive in $74.1 \%$. The sensitivity of these two drugs was found to be $41.5 \%$ and $22 \%$, and $67.6 \%$ and $75.9 \%$ respectively in different studies ${ }^{17,26}$.

This study showed that aminoglycosides (Gentamycin and Amikacin) are effective against pseudomonas. However, Amikacin (91.96\%) was more sensitive than gentamycin $(70.18 \%)$. Amikacin sensitivity pattern was comparable to a local and an international study ${ }^{23-24}$.

We found imipenam to be much effective $(94.48 \%)$ against pseudomonas. The sensitivity of imipenam has been varyingly reported. It was comparable to our study in some cases and even much better in other studies $6,18,25,27,28$. Highest Sensitive of all the drugs was collistin $(100 \%)$ which has also been reported in many studies ${ }^{22,29}$. This shows the extensive resistance pattern of pseudomonas in increasing trend for anti-pseudomonal antibiotics. This is probably due to largest gene sequence and greater number of base pairs of bacteria developing multiple mechanisms of resistance ${ }^{8-10}$.

Our study has highlighted the recent susceptibility pattern for pseudomonas against antimicrobials and its increasing resistance. This has emphasized the need for judicious use of antibiotics especially in hospitalized and immune-compromised patients. However, our study had few limitations. It was a single centre study in which patients' comorbidities were not studied. The details of the settings either medical or surgical were also not included in the study. So, a multicentre study with the details of the patients and the ward is needed in order to have an actual idea of disease burden and to help improve health care facilities.

\section{CONCLUSION}

The Study showed increased resistance and increased frequency of pseudomonas being isolated especially in hospital settings. Amikacin and Imipenam are two effective drugs against pseudomonas. Cautious use of antibiotics and a multicentre study with related details is recommended.

\section{ETHICAL APPROVAL}

The study was approved from Institutional Review Board of King Edward Medical University, Lahore, Pakistan, vide reference No. 333/RC/KEMU, dated May 11, 2020.

\section{REFERENCES}

1- Sengupta S., Chattopadhyay M. K., Grossart, H. P. (2013). The multifaceted roles of antibiotics and antibiotic resistance in nature. Frontiers in microbiology, 4, 47.

2- Ventola, C. L. (2015). The antibiotic resistance crisis: part 1: causes and threats. Pharmacy and therapeutics, 40(4), 277.

3- Dar O. A., Hasan R., Schlundt J., Harbarth S., Caleo G., Dar F. K., et al. (2016). Exploring the evidence base for national and regional policy interventions to combat resistance. The Lancet, 387(10015), 285-295.

4- Castro-Sánchez E., Moore L. S., Husson F., Holmes, A. H. (2016). What are the factors driving antimicrobial resistance? Perspectives from a public event in London, England. BMC infectious diseases, 16(1), 465.

5- Boucher H. W., Talbot G. H., Bradley J. S., Edwards J. E., Gilbert D., Rice L. B., et al (2009). Bad bugs, no drugs: no ESKAPE! An update from the Infectious Diseases Society of America. Clinical infectious diseases, 48(1), 1-12.

6- Al-Zaidi, J. R. (2016). Antibiotic susceptibility patterns of Pseudomonas aeruginosa isolated from clinical and hospital environmental samples in Nasiriyah, Iraq. African journal of microbiology research, 10(23), 844-849.

7- Forestier C., Guelon D., Cluytens V., Gillart T., Sirot J., De Champs, C. (2008). Oral probiotic and prevention of Pseudomonas aeruginosa infections: a randomized, double-blind, placebo-controlled pilot study in intensive care unit patients. Critical Care, 12(3), 1-10.

8- Lambert, P. A. (2002). Mechanisms of antibiotic resistance in Pseudomonas aeruginosa. Journal of the royal society of medicine, 95( $\mathrm{Suppl} 41), 22$.

9- Rafiee R., Eftekhar F., Tabatabaei S. A., Tehrani, D. M. (2014). Prevalence of extended-spectrum and metallo $\beta$-lactamase production in AmpC $\beta$-lactamase producing Pseudomonas aeruginosa isolates from burns. Jundishapur journal of microbiology, 7(9).

10- Tomás M., Doumith M., Warner M., Turton J. F., Beceiro A., Bou G., et al. (2010). Efflux pumps, OprD porin, AmpC $\beta$ lactamase, and multiresistance in Pseudomonas aeruginosa isolates from cystic fibrosis patients. Antimicrobial agents and chemotherapy, 54(5), 2219-2224.

11- Ali Z., Mumtaz N., Naz S. A., Jabeen N., \& Shafique M. (2015). Multi-drug resistant Pseudomonas aeruginosa: a threat of nosocomial infections in tertiary care hospitals. JPMA, 65(12), 12-16.

12- Perez F., Endimiani A., Hujer K. M., Bonomo, R. A. (2007). The continuing challenge of ESBLs. Current opinion in pharmacology, 7(5), 459-469. 
13- Fernández L., Gooderham W. J., Bains M., McPhee J. B., Wiegand I., Hancock, R. E. (2010). Adaptive resistance to the "last hope" antibiotics polymyxin B and colistin in Pseudomonas aeruginosa is mediated by the novel two-component regulatory system ParRParS. Antimicrobial agents and chemotherapy, 54(8), 3372-3382.

14- Dößelmann B., Willmann M., Steglich M., Bunk B., Nübel U., Peter S., et al. (2017). Rapid and consistent evolution of colistin resistance in extensively drugresistant Pseudomonas aeruginosa during morbidostat culture. Antimicrobial agents and chemotherapy, 61(9).

15- Lescat M., Poirel L., Tinguely C., Nordmann, P. (2019). A resazurin reduction-based assay for rapid detection of polymyxin resistance in Acinetobacter baumannii and Pseudomonas aeruginosa. Journal of clinical microbiology, 57(3).

16- Khan F., Khan A., Kazmi, S. U. (2014). Prevalence and susceptibility pattern of multi drug resistant clinical isolates of Pseudomonas aeruginosa in Karachi. Pakistan journal of medical sciences, 30(5), 951.

17- CLSI. Performance Standards for Antimicrobial Disk Susceptibility Tests. 13th ed. CLSI standard M02. Wayne, PA: Clinical and Laboratory Standards Institute; 2018.

18- Sarwar S., Sohail M., Ahmed, M. (2013). Recent trends in antibiotics susceptibility pattern of Pseudomonas sp. isolated from clinical samples of Punjab, Pakistan. Lat Am J Pharm, 32(8), 1244-1248.

19- Saeed W. M., Ghanem S., El Shafey H. M., Manzoor, N. (2018). In vitro antibiotic resistance patterns of Pseudomonas spp. isolated from clinical samples of a hospital in Madinah, Saudi Arabia. African Journal of Microbiology Research, 12(1), 19-26.

20- Abbas S. H., Naeem M., Adil M., Naz S. M., Khan A., Khan, M. U. (2015). Sensitivity patterns of Pseudomonas aeruginosa isolates obtained from clinical specimens in Peshawar. Journal of Ayub Medical College Abbottabad, 27(2), 329-332.

21- Shah S. N., Ullah B., Basit A., Begum A., Tabassum A., Zafar S., et al. (2016). Prevalence and susceptibility patterns of bacteria causing respiratory tract infections in North Waziristan, Pakistan. Pak J Pharm Sci, 29(Suppl 2), 701-706.

22- Farooq L., Memon Z., Ismail M. O., Sadiq, S. (2019). Frequency and antibiogram of multi-drug resistant pseudomonas aeruginosa in a Tertiary Care Hospital of Pakistan. Pakistan journal of medical sciences, 35(6), 1622.
23- Samad A., Ahmed T., Rahim A., Khalil A., Ali I. (2017). Antimicrobial susceptibility patterns of clinical isolates of Pseudomonas aeruginosa isolated from patients of respiratory tract infections in a Tertiary Care Hospital, Peshawar. Pakistan journal of medical sciences, 33(3), 670.

24- Micek S. T., Wunderink R. G., Kollef M. H., Chen C., Rello J., Chastre J., et al. (2015). An international multicenter retrospective study of Pseudomonas aeruginosa nosocomial pneumonia: impact of multidrug resistance. Critical Care, 19(1), 219.

25- Sarwat T., Rashid M., Rastogi V., Chander, Y. (2015). A comparative study of Antibiogram of Pseudomonas aeruginosa in Hospital and community acquired infections. Int J Curr Microbiol App Sci, 1, 286-291.

26- Ahmed O. B. (2016). Incidence and antibiotic susceptibility pattern of Pseudomonas aeruginosa Isolated from Inpatients in Two Tertiary Hospitals. Clinical Microbiology: Open Access.

27- Fatima A., Naqvi S. B., Khaliq S. A., Perveen S., Jabeen, S. (2012). Antimicrobial susceptibility pattern of clinical isolates of Pseudomonas aeruginosa isolated from patients of lower respiratory tract infections. SpringerPlus, 1(1), 70.

28- Sabir R., Alvi S. F. D., Fawwad A., Basit, A. (2014). Antibiogram of Pseudomonas aeruginosa and methicillin-resistant Staphylococcus aureus in patients with diabetes. Pakistan journal of medical sciences, 30(4), 814.

29- Sader H. S., Farrell D. J., Castanheira M., Flamm R. K., \& Jones, R. N. (2014). Antimicrobial activity of ceftolozane/tazobactam tested against Pseudomonas aeruginosa and Enterobacteriaceae with various resistance patterns isolated in European hospitals (2011-12). Journal of antimicrobial chemotherapy, 69(10), 2713-2722.

\section{AUTHOR'S CONTRIBUTIONS}

SZ: Concept and Design, Manuscript writing

HMM: Manuscript writing

HB: Supervision, Data collection, Data analysis

AA: Data collection, Data analysis

HMSJ: Study Design, Critical Review

AS: Data collection, Critical Review 